\title{
Anticancer Potential of Silibinin Loaded Polymeric Nanoparticles against Breast Cancer Cells: Insight into the Apoptotic Genes Targets
}

\author{
Ali Pourgholi ${ }^{1}$, Mehdi Dadashpour ${ }^{2,3 *}$, Akram Mousapour ${ }^{2}$, Akram \\ Firouzi Amandi ${ }^{4}$, Nosratollah Zarghami ${ }^{5,6,7}$.
}

\begin{abstract}
Silibinin (SIL) is a natural polyphenolic flavonoid with multiple biological and anti-cancer features. However, the complex hydrophobic nature and inadequate bioavailability of SIL hinder its efficiency at tumor sites. Investigating the possibility of an extensive strategy for better treatment of breast cancer, we carried out a comparative exploration of the inhibitory effect of SIL and SIL loaded PLGA-PEG nanoparticle (SIL-NPs) on the expression of the proapoptotic target genes, which is considered as an influential molecular target for treatment of breast cancer. The main diameter of SIL-NPs was $220 \pm 6.37$ and $150 \pm 23.14 \mathrm{~nm}$ via DLS and FE-SEM respectively. Furthermore, the zeta potential of PLGA-PEG and SIL-NPs was $-5.48 \pm 0.13$ and $-6.8 \pm 0.26 \mathrm{mV}$ respectively. SIL encapsulation efficiency and drug release were determined by about $82.32 \%$ by analyzing the calibration curve of SIL absorbance at $570 \mathrm{~nm}$. Cytotoxicity of SIL and SIL-NPs was conducted by MTT assay after 24, 48, and $72 \mathrm{~h}$ of exposure times, and the gene expression levels of apoptotic genes, $p 53$ and $h T E R T$ was measured by real-time PCR. Evaluation of drug toxicity revealed that SIL-NPs represents higher cytotoxic effects than pure SIL in a time and dose-dependent manner. Moreover, the results demonstrated that SIL-NPs could induce apoptosis in breast cancer cells by upregulation of caspase-3, caspase-7, p53 and Bax, along with Bcl-2, hTERT, survivin and Cyclin D1 down regulation. Our results indicated that PLGA-PEG can be used as stable carriers in nano-dimensions and SIL-NPs can be considered as a promising pharmacological agent for cancer therapy.
\end{abstract}

Keywords: Silibinin- PLGA-PEG- polymeric nanoparticles- apoptosis- breast cancer

Asian Pac J Cancer Prev, 22 (8), 2587-2596

\section{Introduction}

With the increasing number of cancer-related death worldwide, cancer has become one of the most important health issues and second principal causes of mortality estimated to reach for 606,520 people deaths in the United States in 2020 (national cancer institute) (Nejati-Koshki et al., 2014; Farajzadeh et al., 2018; Pahlavan et al., 2020). Among three prevalent neoplastic disease in women including colorectal cancer, lung cancer, breast cancer accounts for about $30 \%$ of all diagnosed cases and impacts over 1.5 million women each year (Lotfi-Attari et al., 2017; Jeddi et al., 2019; Nikmanesh et al., 2020). Several established risk factors have been reported for breast cancer such as sex, aging, estrogen, family history, gene mutations and unhealthy lifestyle (Maasomi et al.,
2017; Abbasi et al., 2021; Nejati et al., 2021a). In addition, low-risk single nucleotide polymorphisms, mutations of high-or moderate-risk genes e.g., BRCA1, BRCA2, TP53, ATM and CHEK are common factors contributing breast cancer (Cancer, 2019; Du et al., 2019). Indeed, breast cancer is an outcome of changes in cellular processes that promote cell proliferation and metastasis along with suppressing apoptosis (Javan et al., 2019; Pahlavan et al., 2020; Rasouli et al., 2020).

Estrogen receptor (ER) is predominantly overexpressed invasive breast tumors representing an important hallmark for the prophecy and forecast of breast cancer. Numerous variables in tissue processing can influence the ER expression level in breast tumors (Caruana et al., 2020).

The presence of ER in mammalian tumors increases hormone therapy responses by $55 \%$ to $80 \%$. In addition,

${ }^{1}$ Department of Pharmaceutical Chemistry, Faculty of Pharmacy, Eastern Mediterranean University, Famagusta, TR North Cyprus, Turkey. ${ }^{2}$ Stem Cell Research Center, Tabriz University of Medical Sciences, Tabriz, Iran. ${ }^{3}$ Department of Biotechnology, School of Medicine, Semnan University of Medical Sciences, Semnan, Iran. ${ }^{4}$ Department of Immunology, School of Medicine, Tabriz University of Medical Sciences, Tabriz, Iran. ${ }^{5}$ Department of Clinical Biochemistry and Laboratory Sciences, Faculty of Medicine, Tabriz, Iran. ${ }^{6}$ Department of medicine, Faculty of medicine, Istanbul Aydin University, Istanbul, Turkey. ${ }^{7}$ Drug Applied Research Center, Tabriz University of Medical Sciences, Tabriz,Iran.*For Correspondence: zarghami@tbzmed.ac.ir, Dadashpourm@tbzmed.ac.ir 
ER has been reported to be contributed with the cytotoxic effects of flavonoids on breast cancer cells (Wang and Yuan, 2018).

The most common approaches that used today to treatment of cancer include surgery, chemotherapy, radiotherapy and their combination. However, these approaches encounter several hindrances and side effects such as systemic toxicity, drug resistance, high reoccurrence, and various side effects including neutropenia, thrombocytopenia, anemia, nausea, and hair loss (Mohammadian et al., 2017; Mellatyar et al., 2018). In this regard, searching for novel therapeutic agents with natural sources passing higher acceptability and safety along with limited side effects have been greatly taken into the attention (Rasouli and Zarghami, 2018; Faramarzi et al., 2019). Besides, an effective combination of natural agents with currently used chemotherapeutics could result in a synergic inhibitory effect and higher therapeutic effects (Chatran et al., 2018).

Flavonoids are a group of plant derived polyphenolic substances with anti-oxidant possessions that can be exploited in cancer therapy (Tavsan and Kayali, 2019).

SIL $\left(\mathrm{C}_{25} \mathrm{H}_{22} \mathrm{O}_{10}\right)$ is flavonoid extracted from silybum marianum flavinoligan (known as milk thistle), with natural anti-inflammatory properties inhibiting oxidative stress (Dadashpour et al., 2017; Chen et al., 2020). Several studies have indicated the anti-proliferative effect of SIL as well as G1 arrest property, apoptosis induction, and decreasing hTERT expression (Amirsaadat et al., 2010; Abdollahi et al., 2015). Hence, the combination of SIL with current chemotherapeutic agents could enhance the efficiency of chemotherapy both in vivo and in vitro (Gohulkumar et al., 2014). However, the hydrophobic and multiring structure of SIL, as well as limited bioavailability, results in low aqueous solubility of SIL hindering its preclinical and clinical oral administration (Gogoi et al., 2018). In this regard, several approaches have been employed to enhance SIL bioavailability including complexation with soluble derivatives e.g., cyclodextrins, phosphatidylcholine, and phospholipids. Moreover, nanoparticles and nanomicelles are widely used for efficient drug delivery with the aim of side effect reduction (Gogoi et al., 2018; Serati-Nouri et al., 2020; Nejati et al., 2021b).

Nanoencapsulation of hydrophobic drugs represent various advantages in pharmaceutical sciences including controlled and stimuli-responsive drug release, long-term stability, non-toxicity, targeted drug delivery, and beneficial physiological properties (Amirsaadat et al., 2017; Patel et al., 2020). Among all the biomaterials, poly (lactic-co-glycolic acid) known as PLGA is an FDA-approved polymer extensively used in developing NPs due to high biocompatibility, small size, and biodegradability (Hossainzadeh et al., 2019; Norouzi et al., 2019; Samadzadeh et al., 2021). On the other hand, mucus permeability of PLGA is hindered according to hydrophobic interaction with mucin fibers (Mohammadian et al., 2016a; Mohammadian et al., 2016b; Adlravan et al., 2021). In this regard, copolymerization of PLGA with polyethylene glycol (PEG) or "PEGylation" improves cellular penetration and stability of PLGA in complex physiological environments (Dadashpour et al., 2017; Mousazadeh et al., 2020). Nanoencapsulation of hydrophobic components SIL with PLGA-PEG have improved the efficiency of drug delivery and reduced degradation rate in vivoThe aim of the present study was to evaluate anti-cancer and pro-apoptotic effects of SIL and SIL-NPs against T47D and MDA-MB231 breast cancer cells.

\section{Materials and Methods}

\section{Materials}

Glycolide, DL-Lactide, Dimethyl Sulfoxide (DMSO), Dichloromethane (DCM), Poly vinyl alcohol (PVA), Stannous 2-ethylhexanoate and Poly ethylene glycol was obtained from Merck chemical co. (Germany). Sil, were acquired from Sigma-Aldrich. Fetal bovine serum (FBS), Trypsin- EDTA, RPMI 1640, Penicillin G, Streptomycin, Trypsin-EDTA and TRIzol Reagent purchased from Gibco BRL. (Gaithersburg, MD, USA). First Strand cDNA synthesis Kit and SYBR Green PCR Master Mix were purchased from Fermentas (Vilnius, Lithuania).

\section{Methods}

Copolymer synthesis

PLGA-PEG copolymers were synthesized via ringopening polymerization method under vacuum based on previous studies (Duan et al., 2007). For this purpose, lactide and glycolide monomers with the molar ratio of 4:1 in attendance of polyethylene glycol in the molten state were used. Lactide (2.882 g), glycolide ( $0.570 \mathrm{~g})$, and (1.54 g) polyethylene glycol $46.5 \% \mathrm{w} / \mathrm{w}$ were heated to $140^{\circ} \mathrm{C}$ under nitrogen atmosphere in a bottleneck flask containing silicone oil to complete melting. After monomer melting, $\mathrm{Sn}(\mathrm{Oct}) 2$ was added and the reaction mixture was further heated for $1 \mathrm{~h}$, in order to complete polymerization under static vacuum. The synthesized copolymer was dissolved by dichloromethane followed by ice-cold diethyl ether precipitation. Lastly, the physicochemical properties of the product were evaluated by DLS and FE-SEM.

\section{Preparation of SIL-NPS}

SIL was encapsulated into PLGA-PEG copolymer through a slightly modified double emulsion method (W/O) (34). Briefly, $2 \mathrm{mg}$ of SIL and $100 \mathrm{mg}$ of pre-prepared PLGA-PEG copolymer (1:50) was dissolved in $5 \mathrm{ml}$ of dichloromethane and $10 \mathrm{ml}$ of $1 \%$ PVA (polyvinyl alcohol), respectively. The PLGA-PEG mixture was homogenized at 72,000 rpm for 20 minutes using a homogenizer and the PVA-SIL solution was gradually added to the mixture. After complete evaporation of the organic solvent (dichloromethane) by the rotary apparatus for 15 minutes at room temperature, the final w/o emulsion was centrifuged and purified by a $100 \mathrm{kDa}$ cut off amicon filter at $15,000 \mathrm{rpm}$ for 40 minutes to separate the encapsulated SIL. The DCM was completely vaporized using rotary evaporator under low vacuum. Then, two cycles of centrifugation were used to purification of NPs. Finally, the NPs formed washed twice with distilled water, lyophilized, and stored at $20^{\circ} \mathrm{C}$ for further experimentation. 
Physicochemical characterization of SIL-PLGA-PEG

Confirmation of SIL Loading by FTIR

The identification of functional groups in the SIL, PLGA/PEG copolymer and SIL-NPs were analyzed using FTIR spectroscopy (PerkinElmer, Waltham, MA, USA) in the $4,000 \mathrm{~cm}^{-1}$ to $400 \mathrm{~cm}^{-1}$ wavenumber range averaging 100 scans.

\section{NPs morphology}

The shape, surface morphology and microstructure of prepared PLGA-PEG nanoparticles were studied by FE-SEM model Seron Technologies AIS 2100 (Korea). For this, a small quantity of samples was rinsed two times with purified water, freeze-dried, and coated with gold/palladium for scanning electron microscopic observation.

\section{SIL release profile from $N P S$}

Release of SIL from PLGA-PEG copolymer was assayed as follows: $25 \mathrm{mg}$ of SIL-NPs was dissolved in $5 \mathrm{ml} \mathrm{PBS}$ at $\mathrm{pH} 4.4$ and 7.4. Sample were set into a to dialysis membrane tubing (MW cut off: 3,000 ). The dialysis membrane tubing was introduced into vials containing $25 \mathrm{ml}$ of buffer solution. At specific time, intervals from $0.5 \mathrm{~h}$ up to $120 \mathrm{~h}$, SIL released samples were replaced with fresh PBS. Samples were centrifuged and supernatants were collected for UV-Visibility analysis at $570 \mathrm{~nm}$ (maximum absorption wavelength) using BEL LGS 53 spectrophotometer. The percentage of SIL release from NPs was measured by following equation:

\section{$\mathrm{EE} \%=[($ Drug Total - Drug Filtered $) /($ Drug Total $)] \times 100$}

\section{SIL encapsulation efficiency}

For the evaluation of encapsulation efficiency (EE), the freeze-dried drug encapsulated NPs were weighed and dissolved in $\mathrm{H} 2 \mathrm{O}$ : $\mathrm{CH} 3 \mathrm{CN}$ mixture $(50 / 50, \mathrm{v} / \mathrm{v})$ and then extracted with $\mathrm{CH} 3 \mathrm{OH}$. The resulted NPs were filtered using an Amicon $3 \mathrm{kDa}$ centrifugal filter. Few microliters of filtrate were determined by high performance liquid chromatography (HPLC). The encapsulated drug is stated both as drug loading (DL) and entrapment efficiency (EE), characterized by Eqs. (1) and (2), respectively.

$$
\begin{aligned}
& \text { Eq.1 } \mathrm{DL}(\%)=\frac{\text { mass of drug in NPs }}{\text { total NPs mass }} \times 100 \\
& \text { Eq.2 } \quad \mathrm{EE}(\%)=\frac{\text { mass of drug in NPs }}{\text { total mass drug }} \times 100
\end{aligned}
$$

\section{Cell line maintenance}

Two human breast cancer cells, MCF-7 and MDA-MB-231 were cultured in RPMI-1640 media. The media was supplemented with $15 \%$ FBS, streptomycin $(100 \mathrm{mg} / \mathrm{mL})$, and penicillin $(100 \mathrm{U} / \mathrm{mL})$. Cells were incubated at $37^{\circ} \mathrm{C}$ with $15 \% \mathrm{CO}_{2}$ in a humidified atmosphere. All experiments were carried out on logarithmically growing cells.

\section{Cell viability test}

In order to evaluate SIL and SIL-NPs effects on growth of cell, MTT assay was performed on MDA-MB231 and MCF-7 breast cancer cell lines. In this assay, $2 \times 10^{4}$ cells/well were seeded in 96-well plates and cultured. Different concentrations (0-125 $\mu \mathrm{M})$ of SIL and SIL-NPs were added after $24 \mathrm{~h}$ in equivalent doses for three-time intervals $(24,48$, and $72 \mathrm{~h})$. Control wells including cells without drug treatment and cells treated with free NPs were treated with an equivalent amount (1\%) of ethanol. Following the treatment, the media containing SIL, SIL-NPs, and controls were eliminated closely and $200 \mu \mathrm{L}$ of $2 \mathrm{mg} / \mathrm{mL}$ MTT dissolved in PBS was supplemented to each well, and the plates were coated with aluminum foil to incubate at $37^{\circ} \mathrm{C}$ for 4 hours with a $5 \% \mathrm{CO}_{2}$ atmosphere. Thereafter, the contents of all each well were removed and $200 \mu \mathrm{l}$ of DMSO mixed by pipette and left for $45 \mathrm{sec}$. Finally, the optical density (OD) was read with multi-well microplate reader (ELIZA reader, Organon Teknika).

\section{Real-time PCR assay}

RT-PCR assay was conducted to assess expression of P53, caspase 3, 7, cyclin D1, survivin, Bax, Bcl2, and $h T E R T$ genes under free and nanoformulated of SIL treatment. After $24 \mathrm{~h}$ drug exposure, cells were used for RNA extraction and complementary DNA (cDNA) synthesis using RNX-Plus and Prime Script RT reagent kits respectively. The pureness and concentration of total RNA were assessed by Thermo Scientific ${ }^{\mathrm{TM}}$ NanoDrop ${ }^{\mathrm{TM}}$ based on OD260/280 ratio measurements. cDNA synthesis was performed according to the protocol using $2 \mu \mathrm{g}$ of pure RNA as a template. Levels of hTERT gene expression was determined in a total volume of $14 \mathrm{~mL}$ per reaction using a Real-Time PCR Master Mix (BioFACT). Real-time PCR mixture contained SYBR Green qPCR Master Mix $2 x(7 \mu \mathrm{L})$, a mixture of forward and reverse primers $(0.3 \mu \mathrm{L})$, diluted cDNA $(1 \mu \mathrm{L})$, and $\mathrm{H}_{2} \mathrm{O}$ PCR grade $(4.7 \mu \mathrm{L})$. The amplification condition was set as the following: preincubation for $15 \mathrm{~min}$ at $95^{\circ} \mathrm{C}, 45$ cycles of denaturation for $10 \mathrm{~s}$ at $95^{\circ} \mathrm{C}$, annealing for $30 \mathrm{~s}$ at $58^{\circ} \mathrm{C}$ and extension for $20 \mathrm{~s}$ at $72^{\circ} \mathrm{C}$, which was followed by a melting curve analysis step. The efficiency of real-time PCR for targeted genes was relatively determined and normalized by the housekeeping GAPDH gene. The sequences of the forward and reverse primers are listed in Table 1. Each reaction was done in triplicate and the comparative Livak method $\left(2^{-\Delta \Delta C T}\right)$ was employed for data analysis.

\section{Statistical analysis}

Graph Pad Prism version 7.01 was used for statistical analyses of data. Data are presented as mean \pm standard deviation and the difference between groups were determined by one-way analysis of variance (ANOVA). All experiments were performed in triplicate to assess reproducibility. $\mathrm{P}$-values of $\mathrm{p}<0.05$ was considered as statistically significant. 
Table 1. Primer Sequences Used in Real-Time PCR Technique

\begin{tabular}{|c|c|c|}
\hline Genes & Primer Sequence & PCR product size $(b p)$ \\
\hline \multirow[t]{2}{*}{ hTERT } & F: 5'-CCCATTTCATCAGCAAGTTTGG-3' & 94 \\
\hline & R: 5'-CTTGGCTTTCAGGATGGAGTAG-3' & \\
\hline \multirow[t]{2}{*}{$G A P D H$} & F: 5'-CATGAGAAGTATGACAACAGCCT-3' & 113 \\
\hline & R: 5'-AG TCCTTCCACGATACCAAAGT-3' & \\
\hline \multirow[t]{2}{*}{$b c l-2$} & F: 5' GATGTGATGCCTCTGCGAAG -3' & 93 \\
\hline & R: 5'-CATGCTGATGTCTCTGGAATCT-3' & \\
\hline \multirow[t]{2}{*}{$B a x$} & F: 5'-GGTTGTCGCCCTTTTCTA-3' & 98 \\
\hline & R: 5'- CGGAGGAAGTCCAATGTC -3' & \\
\hline \multirow[t]{2}{*}{ caspase-3 } & F: 5'- TTCAGAGGGGATCGTTGTAGAAGTC -3' & 178 \\
\hline & R: 5'- CAAGCTTGTCGGCATACTGTTTCAG -3' & \\
\hline \multirow[t]{2}{*}{ caspase-7 } & F: 5'-GGACCGAGTGCCCACTTATC-3' & 149 \\
\hline & R: 5'-TCGCTTTGTCGAAGTTCTTGTT-3' & \\
\hline \multirow[t]{2}{*}{ Cyclin D1 } & F: 5'-AGACCTTCGTTGCCCTCTGT-3' & 181 \\
\hline & R: 5'-CAGTCCGGGTCACACTTGAT-3' & \\
\hline \multirow[t]{2}{*}{ Survivin } & F: 5' GATGTGATGCCTCTGCGAAG -3' & 103 \\
\hline & R: 5'-CATGCTGATGTCTCTGGAATCT-3' & \\
\hline \multirow[t]{2}{*}{$P 53$} & F: 5'- GACGGTGACACGCTTCCCTGGATT -3' & 120 \\
\hline & R: 5'- GGGAACAAGAAGTGGAGAATGTCA -3' & \\
\hline
\end{tabular}

\section{Results}

Characterization of SIL- NPS

SIL- NPs size, zeta potential, and morphology

Table 2 summarized the size distribution, zeta potential, and polydispersity index of PLGA-PEG and SIL-NPs, measured by the DLS technique. PLGA-PEG can be emulsified in aqueous solution to form NPs. The size of PLGA-PEG copolymers must be small enough to prevent degradation by the reticuloendothelial system and persist in the circulatory system to be targeted appropriately. DLS analysis had revealed the average size of PLGA-PEG NPs equal to $220 \mathrm{~nm}$ with uniform dispersion while SIL-NPs represented an increased average size of $180 \mathrm{~nm}$ with a range between 150 and 220 $\mathrm{nm}$ indicating the SIL loading within PLGA-PEG core (Figure 1). Zeta potential analysis indicated information of the surface charge and high stability of the NPs. The appropriate surface charge of PLGA-PEG NPs permits their easy absorption through negatively charged

Table 2. Mean $( \pm \mathrm{SD})$ Particle Diameter, Polydispersity $( \pm \mathrm{SD})$ and Zeta Potential of Drug Loaded PLGA-PEG NPs.

\begin{tabular}{lccccc}
\hline Formulation & $\begin{array}{c}\text { Particle size } \\
(\mathrm{nm}) \mathrm{a}\end{array}$ & $\begin{array}{c}\text { Polydispersity } \\
\text { index }\end{array}$ & $\begin{array}{c}\text { Zeta potential } \\
(\mathrm{mV}) \mathrm{a}\end{array}$ & $\begin{array}{c}\text { Formulation } \\
\text { (nm)a }\end{array}$ & $\begin{array}{c}\text { Particle size } \\
\text { Polydispersity } \\
\text { index }\end{array}$ \\
\hline PLGA/PEG NPs & $180 \pm 2.53$ & 0.125 & $-5.48 \pm 0.13$ & PLGA/PEG NPs & $180 \pm 2.54$ \\
SIL-loaded PLGA/PEG NPs & $220 \pm 6.37$ & 0.136 & $-6.8 \pm 0.26$ & SIL-loaded PLGA/PEG NPs & $220 \pm 6.38$ \\
\hline
\end{tabular}
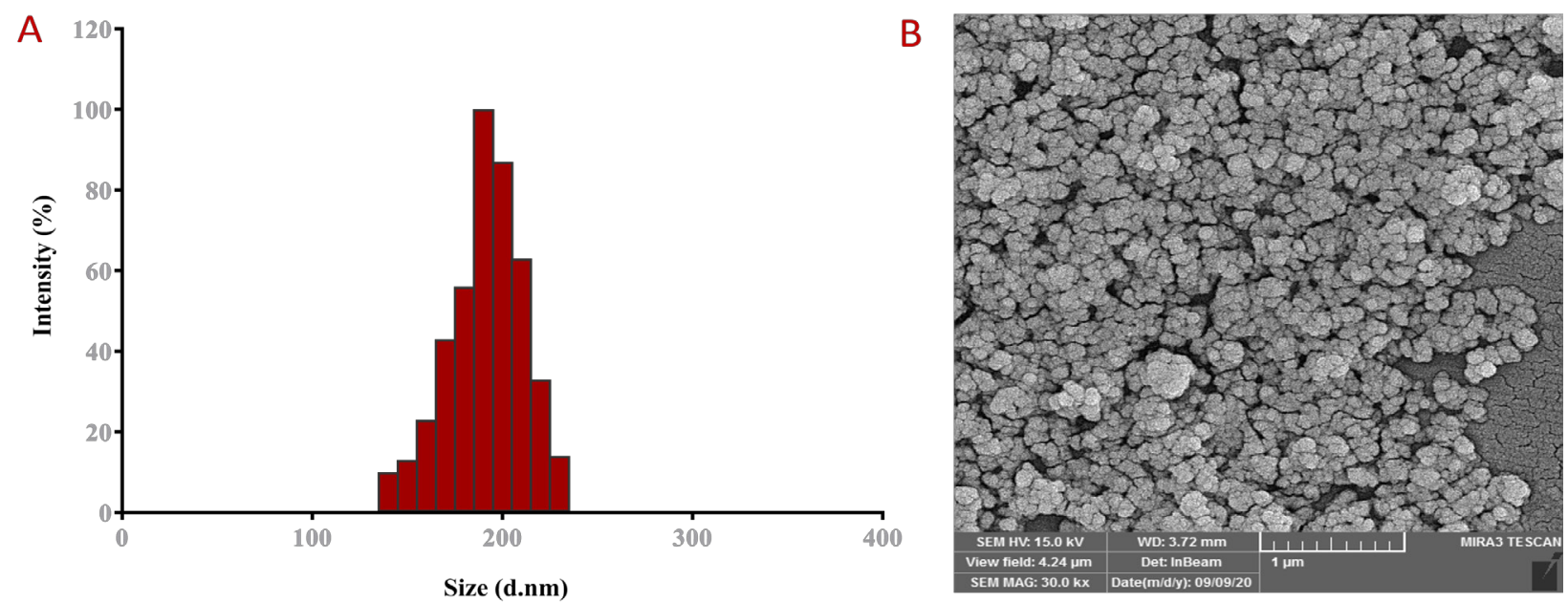

Figure 1. A DLS Histogram Showing the Size Distribution of SIL NPs. The average size ranged from 150-220 nm. B Field emission scanning electron microscopy (FE-SEM) image of surface morphology of SIL NPs 


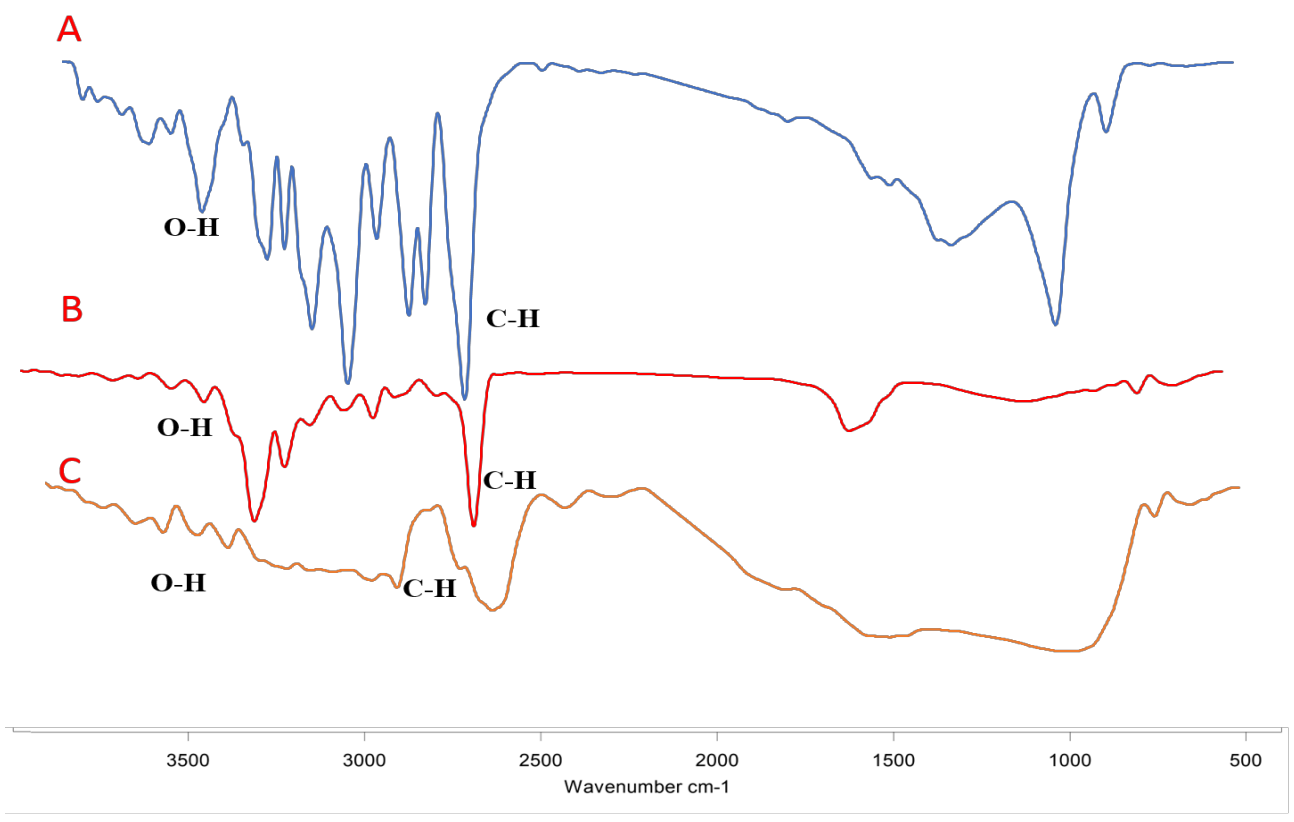

Figure 2. Infrared Spectra of SIL, PLGA/PEG and SIL-NPs

cell membranes and which is contributed to efficient intracellular trafficking and would be useful for more application of PLGA-PEG in vitro studies. Encapsulation of SIL with PLGA-PEG has resulted in the increased surface charge of PLGA-PEG indicating the good stability of the SIL-PLGA-PEG NPs leading to better repulsion

Table 3. IC $\mathrm{IC}_{50}$ Values for the Drug Formulations against MDA-MB-231and MCF-7 Breast Cancer Cell Lines after 24, 48, $72 \mathrm{~h}$ Incubation Time

\begin{tabular}{lcccc}
\hline time & \multicolumn{2}{c}{ MDA-MB-231 } & \multicolumn{2}{c}{ MCF-7 } \\
$(\mathrm{h})$ & SIL $(\mu \mathrm{M})$ & SIL- NPs & SIL $(\mu \mathrm{M})$ & SIL- NPs \\
\hline $24 \mathrm{~h}$ & 65.11 & 55.23 & 53.68 & 48.21 \\
$48 \mathrm{~h}$ & 55.29 & 48.35 & 47.41 & 43.51 \\
$72 \mathrm{~h}$ & 45.11 & 39.54 & 27.14 & 19.13 \\
\hline
\end{tabular}

between the charged particles and prevents aggregation. SEM image revealed that the NPs possess homo-dispersed spherical morphology with a variable size in the range of $150+23.9 \mathrm{~nm}$ (Figure 1).

\section{FTIR studies}

To further confirm SIL loading on designed NPs, FTIR spectroscopy was employed to illustrate the structure of PLGA-PEG NPs and SIL-loaded PLGA-PEG NPs (Figure 2). The FT-IR spectrum of free SIL represented an absorption band including $834 \mathrm{~cm}^{-1}$ related to $\mathrm{C}-\mathrm{O}-\mathrm{C}$ stretching, $1,277 \mathrm{~cm}^{-1}$ for aromatic C-O stretching, 1,464 and $1,512 \mathrm{~cm}^{-1}$ related to the skeleton vibration of aromatic $\mathrm{C}=\mathrm{C}$ ring stretching, $1940 \mathrm{~cm}^{-1}$ demonstrating the $\mathrm{C}=\mathrm{O}$ stretching, 2,854 to 2,925, and $3,431 \mathrm{~cm}^{-1}$ for $\mathrm{C}-\mathrm{H}$ stretching. FT-IR spectrum of the shows the

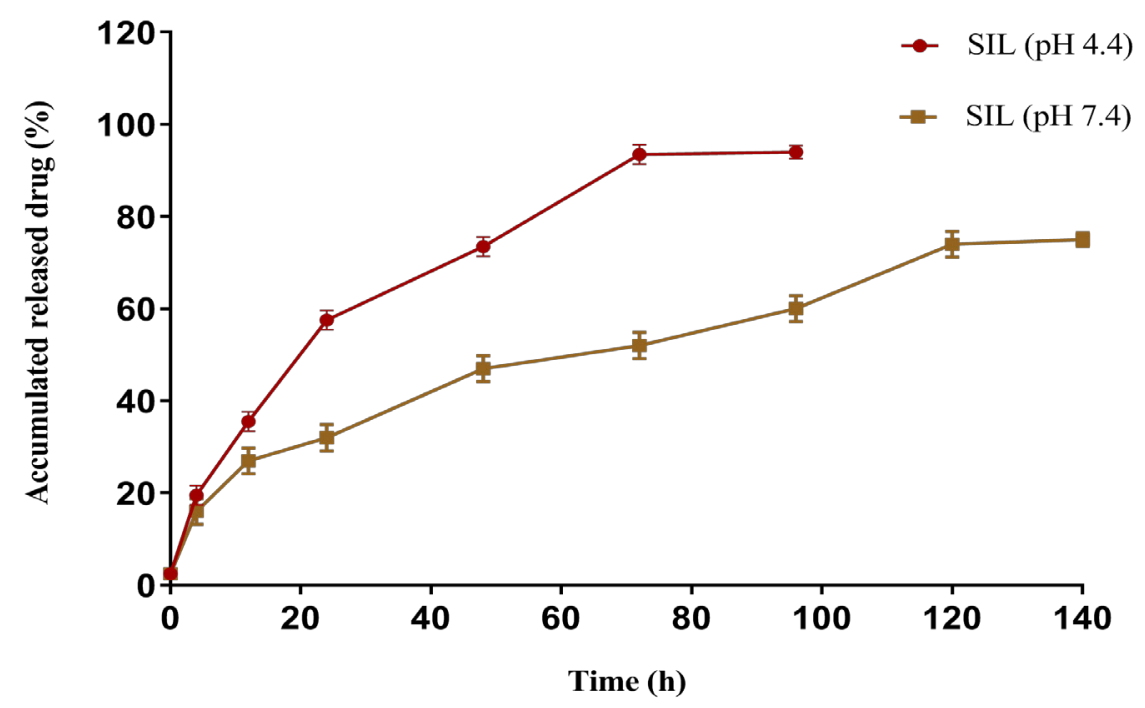

Figure 3. Cumulative Release (\%) Behavior of SIL from NPs in Phosphate Buffered Saline (pH 7.4 and pH 4.4) 
MDA-MB-231

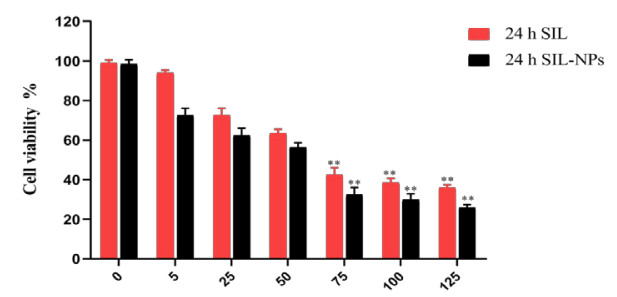

SIL Concentration $(\mu M)$
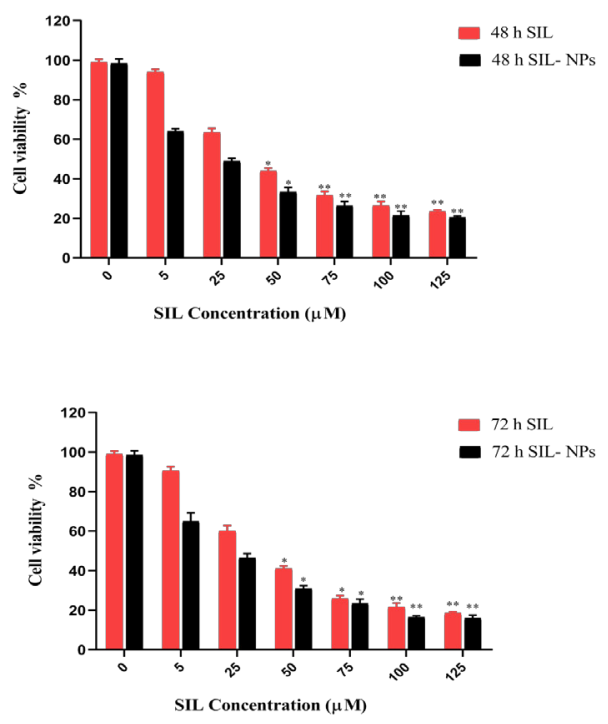

MCF-7
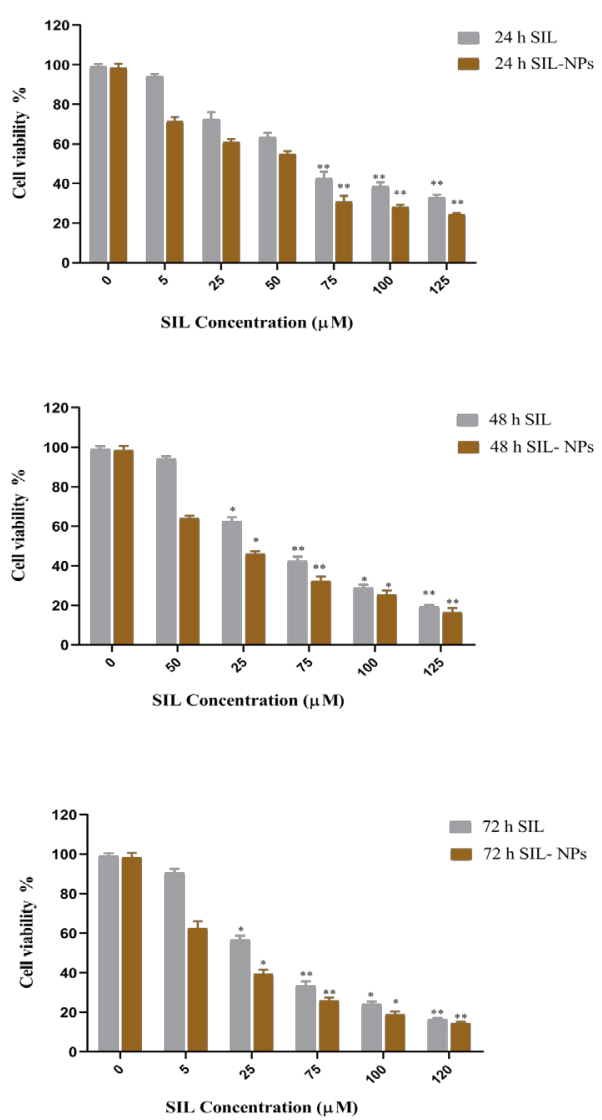

Figure 4. The In Vitro Cytotoxicity by MTT Assay. The viability of MDA-MB-231 and MCF-7 breast cancer cells receiving various treatments SIL and SIL-NPs. Error bars indicate standard deviations. $(* \mathrm{P}$ value $<0.05$, $* *$ P value $<0.001)$.

SIL-PLGA-PEG copolymers represented characteristic peaks at $2880.71 \mathrm{~cm}^{-1}$ and $1768.33 \mathrm{~cm}^{-1}$ which were related to the $\mathrm{C}-\mathrm{H}$ and $\mathrm{C}=\mathrm{O}$ bands stretching, respectively.

\section{In vitro drug release profile}

The release of SIL from PLGA-PEG nanoparticles was performed by dialysis against PBS at the physiological temperature of $37^{\circ} \mathrm{C}$ during a period of $168 \mathrm{hr}$. The release pattern of SIL from PLGA-PEG in $\mathrm{pH}=7.4$ and 4.4 is indicated in (Figure 3). Results demonstrated that a burst increase in SIL release occurs within 3 hours at a $\mathrm{pH}$ of 7.4 , followed by a controlled release of SIL in 6 days. After $120 \mathrm{hr}$, approximately $75 \%$ of the SIL is released from the copolymer at $\mathrm{pH}=7.4$.
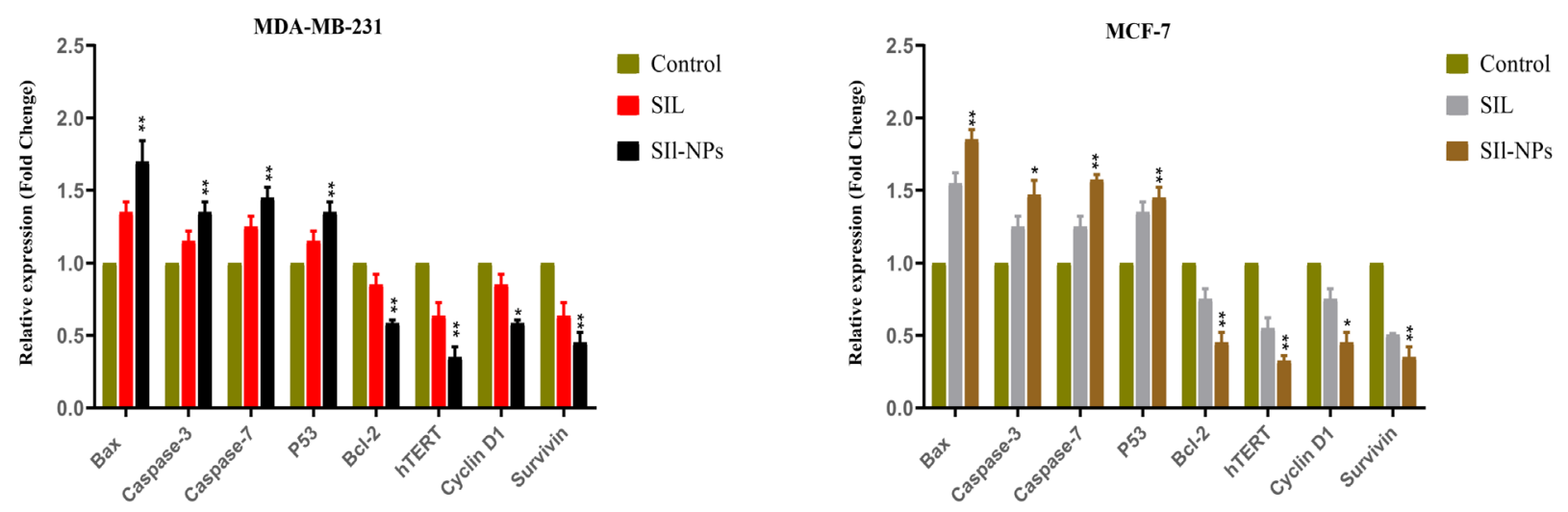

Figure 5. Relative mRNA Expression Levels of P53, Caspase 3, 7, Cyclin D1, Survivin and hTERT Genes in MDAMB-231 and MCF-7 Breast Cancer Cell Line Treated with by Free and Nanoformulated form of Sil. $* \mathrm{P}<0.05, * * \mathrm{P}<$ 0.01 and $* * * \mathrm{P}<0.001$ vs. control was considered significant 


\section{SIL encapsulation efficiency}

The encapsulation efficiencies of SIL in the NPs were $82.32 \%$ at a copolymer/drug with a loading capacity of $11.1 \pm 3.2 \%$, respectively. Moreover, at higher concentrations of SIL, the DL and EE were initiated to be decreasing.

\section{Cytotoxicity studies}

To evaluate the growth inhibition effect of SIL and SIL-NPs, MTT assay was applied using different doses $(0-125 \mu \mathrm{M})$ of free SIL and SIL-NPs on MCF-7 and MDA-MB231 breast cancer cells during 24, 48, and $72 \mathrm{~h}$ (Table 3). Cells without treatment were used as control groups. Table 2 shows the half maximal inhibitory concentration $\left(\mathrm{IC}_{50}\right)$ values of SIL and SIL-NPs against MCF-7 and MDA-MB231 after treatment. Data analysis of MTT assay revealed the cytotoxicity effect of SIL in both free and nanocapsulated forms in a dose- and time- dependent manner. Regarding the diagrams of cytotoxicity analysis displayed in (Figure 4), SIL-NPs possess greater growth inhibitory effect than free SIL with a drastic reduction in $\mathrm{SIL} \mathrm{IC}_{50 \mathrm{~s}}$. In addition, the cytotoxicity of SIL-PLGA-PEG on MCF-7 cells was much more than on MDA-MB231. Treatment of both MDA-MB231 and MCF-7 with equal concentrations of SIL-NPs at certain times, have demonstrated that growth inhibitory effect of SIL-NPs represent a significant difference on MCF-7 compared with MDA-MB23. The results suggested that cytotoxicity of SIL-NPs is more effective in estrogen receptor positive breast cancer cells.

\section{Gene expression findings}

The expression of P53, caspase 3, 7, cyclin D1, survivin, Bax, Bcl-2 and hTERT in MCF-7 and MDA-MB-231 cancer cell lines after exposure to SIL and SIL-NPs for 48 of incubation was determined by real-time PCR. As our results showed, caspase-3, caspase-7, P 53 and Bax (apoptosis markers) mRNA levels were meaningfully increased in the cells treated with nanoformulated forms of SIL compared to control. The decreased RNA transcription level of anti-apoptotic hTERT, Cyclin D1, Survivin and $B c l-2$ genes was observed in free and nanoformulated group compared with untreated cells.

\section{Discussion}

The wide spectrum of cancer chemopreventive ability of SIL has been proved in several cases. SIL inhibits the proliferation and invasion of tumor cells in various cancers. It has been reported that SIL inhibits the invasion and proliferation of hepatocarcinoma cell lines via the induction of program cell death and cell cycle arrest (Boojar et al., 2016). Previous studies found that SIL inhibits ERK1/2 signaling, which indicates that the ERK1/2 signaling pathway may play as an upstream regulator that modulates the SIL-induced Bim signaling pathway. In addition, the effect of SIL on the prohibition of angiogenesis in hepatic cancers is associated with reduced secretion of vascular endothelial growth factor, CD34, and metalloproteinase-2 in a dose dependent manner (Li and Wang, 2016; Zappavigna et al., 2019).
Likewise, high amounts of SIL in human colorectal mucosa was found to be deliverable through ingestion of nontoxic doses of SIL considered to be beneficial in early stages of colon tumorigenesis. SIL can potently hinder the proliferation of LoVo and HT-29 cells both in xenograft and vitro models through the induction of G1 and G2-M cell cycle arrest (Nafees et al., 2018). This effect is incorporated with lower levels of cell division cycle 25C (cdc25C), cyclins (A, B1, D1, D3, and E), and Cdc2/ p34 as well as diminished activity of cyclin-dependent kinases (Errico et al., 2010). SIL is also proved to have the ability to induce program cell death through activation of caspases 3 and 9 along (Zhang et al., 2018). Moreover, SIL treatment decreased the growth and invasiveness of renal cancer 786-O cell while restraining the growth of cell and inducing program cell death in cancer Caki-1 cells through prevention of EGF, ERK1/2 (Raina et al., 2016). In accordance with these findings, several studies have demonstrated the growth inhibitory effects SIL in rat models of urinary bladder cancer, associated with elevated levels of p53 expression, phosphorylation of ERK1/2 and phospho-p65, as well as downregulation of survivin, cyclin D1 (Barros et al., 2020). The use of Sil in breast cancer cells has caused cell apoptosis in MDA-MB-231 and MCF-7 cells, which synergized with inhibition of metastasis through several proteins including insulin growth factor receptor (Si et al., 2020). SIL decreased the expression of 12-O-tetradecanoylphorbol-13-acetateinduced MMP-9, EGFR ligand-induced CD44, and VEGF in a dose-dependent manner. Consistent with the results, SIL enhanced program cell death and stimulated G2-M cell cycle arrest in A2780/taxol cells associated with the downregulation of P-glycoproteins and survivin (Zheng et al., 2017). Synergistic Anti-proliferative ability of SIL in combination with other chemotherapeutics such as Doxorubicin, Metformin (MET), and Chrysin have been greatly assessed. Maasomi et.al, have evaluated the growth inhibitory effects of SIL and chrysin through regulation of hTERT and cyclin D1 in T47D breast cancer cells. Cell viability analysis have demonstrated that Chrysin or SIL can individually prevent the proliferation of T47D, and combination of these compounds resulted in synergistically growth inhibition (Maasomi et al., 2017). Chatran et al., (2018) have evaluated anticancer properties of SIL in mixture with MET on T47D cancer cells. In this study, gene expression analysis represented that in addition to individual drugs, co-delivery of SIL and MET synergistically decreased the cyclin D1 and hTERT expression levels. On the other hand, a plethora of investigations have focused on nanocapsulation of SIL in order to increase bioavailability and efficient delivery of this flavonoid. As a proof of concept, Amirsaadat et al., (2017) loaded SIL in magnetic PLGA-PEG-Fe3O4 NPs to evaluate the expression of hTERT and proliferation of A549 cancer cells. According to this investigation, in comparison with pure SIL, SIL loaded magnetic NPs reduced the hTERT expression more efficiently. Likewise, Amirsaadat et al., (2017) co-loaded SIL and MET on PLGA-PEG nanoparticles to evaluate its effect on the expression levels of apoptosis related and hTERT genes in T47D cancer cells. According to the results obtained, 
MET-SIL NPs demonstrated the more synergistic inhibitory effect on T47D cells growth. In the presented study, we aimed to evaluate cytotoxicity effect of SIL -NPs copolymer on MCF-7 and MDA-MB-231 breast cancer cell lines, compared based on estrogen receptor status. We assessed physicochemical properties of SIL-NPs as well as drug encapsulation efficiency and drug release profile. In addition, the effect of SIL and SIL-NPs on the expression of P53, caspase 3, 7, cyclin D1, survivin and hTERT was determined. In the current study, we synthesized PLGA-PEG with an average size of $220 \mathrm{~nm}$ based on the ring-opening melt polymerization method. SIL was encapsulated with an efficiency of about $83 \%$. The chemical structure of SIL-PLGA-PEG was identified with FTIR and DLS analysis. DLS analysis confirmed that the NP was synthesized in the appropriate size. Furthermore, FE-SEM analysis revealed that SIL-PLGA-PEG possesses a much smaller particle size than those identified by DLS $(180-250 \mathrm{~nm})$. The profile of SIL release from PLGA-PEG was investigated by dialysis at 7.4 and 4.4. It was found that an instantaneous increase in release occurs within 3 hours at a pH of 7.4, which is followed by 6 days of controlled release of SIL resulted in about $88 \%$ of SIL release. The SIL released at $\mathrm{pH} 4.4$, which is a simulation of the acidic state of lysosomes, occurs significantly faster than in $\mathrm{pH} 7.4$ so that about $88 \%$ of the total SIL content was released within 6 days. The sustained drug discharge characteristic of PLGA-PEG lead to the fact that these NPs can be applied as an impressive vehicle for delivery of SIL. MTT assay represented that SIL and SIL-PLGAPEG have cytotoxicity effects on both MCF-7 and MDAMB231cell lines in a time- and dose-dependent manner. Real-time analysis has indicated that in comparison with pure SIL, nanocapsulated SIL represented a greater inhibitory effect on the expression of the hTERT, Cyclin D1, Bcl-2 and survivin genes. Moreover, our results showed that SIL by upregulation of pro-apoptotic genes such as caspase 3, caspase 7 and P53 induced apoptosis in cancerous cells. It appears that SIL-NPs can considerably trigger the downregulation and upregulation of apoptosis related and cell cycle control genes expression in breast cancer cell lines resulting in the inhibition of growth and proliferation which finally leads to cell death by apoptosis.

In conclusion, our findings revealed that PLGA-PEG NPs as a suitable and appropriate nanocarrier may preserve the drug and expose it inside the cells effectively. Moreover, SIL has strong anti-proliferative and anticancer effects with less side effects on MCf-7 and MDA-MB231 cell lines. According to this study, PLGA/PEG NPs prepared by w/o/w emulsion technique and Sil encapsulated into PLGA/PEG NPs to improves drug delivery system in order to develop novel and effective drug delivery systems to fight breast malignant tumor. The results have shown SIL in pure and nano form change and altered the expression level of P53, caspase 3, 7 and cyclin D1, survivin and hTERT. Particularly, the nano-coencapsulated state of SIl had a greater effect on the proapoptotic and antiapoptotic gnes. This preliminary study showed that the loading of the chemotheraputic molecules into PLGA/PEG might lead to develop a novel and safe nanodrug delivery systems for the effective treatment of breast cancer.

\section{Author Contribution Statement}

The authors confirm contribution to the paper as follows: study conception and design: NZ, MD; data collection: AP,AM; analysis and interpretation of results: AF, MD; draft manuscript preparation: MD, AF, NZ. All authors reviewed the results and approved the final version of the manuscript.

\section{Acknowledgments}

The authors thank the Department of Medical Biotechnology, and Medical Nanotechnology, Faculty of Advanced Medical Science of Tabriz University, for all support provided.

\section{Conflict of Interest}

The authors declare that they have no competing interests.

\section{References}

Abbasi A, Dadashpour M, Alipourfard I (2021). Calculation of radium-223 and actinium-225 $\alpha$-emitter radiopharmaceuticals dose rates in treatment of metastatic castration-resistant prostate cancer. J Cancer Res Ther, 17, 348.

Abdollahi P, Ebrahimi M, Motamed N, Samani FS (2015). Silibinin affects tumor cell growth because of reduction of stemness properties and induction of apoptosis in 2D and 3D models of MDA-MB-468. Anticancer Drug Discov, 26, 487-97.

Adlravan E, Nejati K, Karimi MA, et al (2021). Potential activity of free and PLGA/PEG nanoencapsulated nasturtium officinale extract in inducing cytotoxicity and apoptosis in human lung carcinoma A549 cells. J Drug Deliv Sci Technol, 61, 102256.

Amirsaadat S, Jafari-Gharabaghlou D, Alijani S, et al (2021). Metformin and Silibinin co-loaded PLGA-PEG nanoparticles for effective combination therapy against human breast cancer cells. J Drug Deliv Sci Technol, 61, 102107.

Amirsaadat S, Pilehvar-Soltanahmadi Y, Zarghami F, et al (2017). Silibinin-loaded magnetic nanoparticles inhibit hTERT gene expression and proliferation of lung cancer cells. Artif Cells Nanomed Biotechnol, 45, 1649-56.

Barros TM, Lima AP, Almeida TC, et al (2020). Inhibition of urinary bladder cancer cell proliferation by silibinin. Environ Mol Mutagen, 61, 445-55.

Boojar MMA, Hassanipour M, Mehr SE, et al (2016). New aspects of silibinin stereoisomers and their 3-O-galloyl derivatives on cytotoxicity and ceramide metabolism in Hep G2 hepatocarcinoma cell line. Iran J Pharm Res, 15, 421.

Cancer CGoHFiB (2019). Type and timing of menopausal hormone therapy and breast cancer risk: individual participant meta-analysis of the worldwide epidemiological evidence. Lancet, 394, 1159-68.

Caruana D, Wei W, Martinez-Morilla S, et al (2020). Association between low estrogen receptor positive breast cancer and staining performance. NPJ Breast Cancer, 6, 1-6.

Chatran M, Pilehvar-Soltanahmadi Y, Dadashpour M, et al. Synergistic anti-proliferative effects of metformin and silibinin combination on T47D breast cancer cells via hTERT and cyclin D1 inhibition. Drug Res, 68, 710-6.

Chen J, Li D-L, Xie L-N, et al (2020). Synergistic 
anti-inflammatory effects of silibinin and thymol combination on LPS-induced RAW264. 7 cells by inhibition of NF- $\mathrm{KB}$ and MAPK activation. Phytomedicine, 78, 153309.

Dadashpour M, Pilehvar-Soltanahmadi Y, Zarghami N, et al (2017). Emerging importance of phytochemicals in regulation of stem cells fate via signaling pathways. Phytother Res, 31, 1651-68.

Dadashpour M, Yaghoutrang M, Zarghami M, et al (2020). Metformin-loaded nanostructured Plga: A New Strategy for Enhancing Efficacy of Metformin in Breast Cancer Treatment. EURAS J Health, 37.

Du Y, Lin Y, Yin K, et al (2019). Single nucleotide polymorphisms of let-7-related genes increase susceptibility to breast cancer. Am J Transl Res, 11, 1748.

Duan Y, Zhang Y, Gong T, Zhang Z (2007). Synthesis and characterization of MeO-PEG-PLGA-PEG-OMe copolymers as drug carriers and their degradation behavior in vitro. J Mater Sci Mater Med, 18, 2067-73.

Errico A, Deshmukh K, Tanaka Y, et al (2010). Identification of substrates for cyclin dependent kinases. Adv Enzym Regul, 50, 375-99.

Ensign LM, Tang BC, Wang Y-Y, et al (2012). Mucus-penetrating nanoparticles for vaginal drug delivery protect against herpes simplex virus. Sci Transl Med, 4, 138ra179.

Farajzadeh R, Pilehvar-Soltanahmadi Y, Dadashpour M, et al (2018). Nano-encapsulated metformin-curcumin in PLGA/ PEG inhibits synergistically growth and hTERT gene expression in human breast cancer cells. ArtifCells Nanomed Biotechnol, 46, 917-25.

Faramarzi L, Dadashpour M, Sadeghzadeh H, Mahdavi M, Zarghami N (2019). Enhanced anti-proliferative and proapoptotic effects of metformin encapsulated PLGA-PEG nanoparticles on SKOV3 human ovarian carcinoma cells. Artif Cells Nanomed Biotechnol, 47, 737-46.

Gogoi P, Das MK, Ramteke A, Maji TK (2018). Soy flour-ZnO nanoparticles for controlled release of silibinin: Effect of $\mathrm{ZnO}$ nanoparticle, surfactant, and cross-linker. Int J Polym Mater, 67, 543-52.

Gohulkumar M, Gurushankar K, Prasad NR, Krishnakumar N (2014). Enhanced cytotoxicity and apoptosis-induced anticancer effect of silibinin-loaded nanoparticles in oral carcinoma (KB) cells. Mater Sci Eng C, 41, 274-82.

Hossainzadeh S, Ranji N, Naderi Sohi A, Najafi F (2019). Silibinin encapsulation in polymersome: A promising anticancer nanoparticle for inducing apoptosis and decreasing the expression level of miR-125b/miR-182 in human breast cancer cells. $J$ Cell Physiol, 234, 22285-98.

Javan N, Khadem Ansari MH, Dadashpour M, et al (2019). Synergistic antiproliferative effects of co-nanoencapsulated curcumin and chrysin on mda-mb-231 breast cancer cells through upregulating mir-132 and mir-502c. Nutr Cancer, 71, 1201-13.

Li W-G, Wang H-Q (2016). Inhibitory effects of Silibinin combined with doxorubicin in hepatocellular carcinoma; an in vivo study. $J$ BUON, 21, 917-24.

Lotfi-Attari J, Pilehvar-Soltanahmadi Y, Dadashpour M, et al (2017). Co-delivery of curcumin and chrysin by polymeric nanoparticles inhibit synergistically growth and hTERT gene expression in human colorectal cancer cells. Nutr Cancer, 69, 1290-9.

Maasomi ZJ, Soltanahmadi YP, Dadashpour M, et al (2017). Synergistic anticancer effects of silibinin and chrysin in T47D breast cancer cells. Asian Pac J Cancer Prev, 18, 1283.

Mellatyar H, Talaei S, Pilehvar-Soltanahmadi Y, et al (2018). 17-DMAG-loaded nanofibrous scaffold for effective growth inhibition of lung cancer cells through targeting HSP90 gene expression. Biomed Pharm, 105, 1026-32.
Mohammadian F, Pilehvar-Soltanahmadi Y, Alipour S, Dadashpour M, Zarghami N (2017). Chrysin alters microRNAs expression levels in gastric cancer cells: possible molecular mechanism. DrugRes, 67, 509-14.

Mousazadeh H, Pilehvar-Soltanahmadi Y, Dadashpour M, Zarghami N (2020). Cyclodextrin based natural nanostructured carbohydrate polymers as effective non-viral siRNA delivery systems for cancer gene therapy. J Control Release, 2020.

Nafees S, Mehdi SH, Zafaryab M, et al (2018). Synergistic interaction of rutin and silibinin on human colon cancer cell line. Arch Med Res, 49, 226-34.

Nejati K, Alivand M, Arabzadeh A (2021). MicroRNA-22 in female malignancies: focusing on breast, cervical, and ovarian cancers. Pathol Res Pract, 2021, 153452.

Nejati K, Dadashpour M, Gharibi T, Mellatyar H, Akbarzadeh A (2021). Biomedical applications of functionalized gold nanoparticles: A Review. J Clust Sci, 2021, 1-16.

Nejati-Koshki K, Akbarzadeh A, Pourhassan-Moghaddam M (2014). Curcumin inhibits leptin gene expression and secretion in breast cancer cells by estrogen receptors. Cancer Cell Int, 14, 1-7.

Norouzi M, Yasamineh S, Montazeri M, et al (2019). Recent advances on nanomaterials-based fluorimetric approaches for microRNAs detection. Mater Sci Eng C, 104, 110007.

Pahlavan Y, Nasr MM, Abdolahinia ED, et al (2020). Prominent roles of microRNA-142 in cancer. Pathol Res Pract, 2020, 153220

Patel P, Raval M, Sheth N (2020). Silibinin loaded solid lipid nanoparticles: Effect of Different Lipids and Surfactants on Physicochemical Properties of Nanoparticle. Int J Pharm Invest, 10, 332-8.

Raina K, Kumar S, Dhar D, Agarwal R (2016). Silibinin and colorectal cancer chemoprevention: a comprehensive review on mechanisms and efficacy. J Biomed Res, 30, 452.

Rasouli S, Montazeri M, Mashayekhi S, et al (2020). Synergistic anticancer effects of electrospun nanofiber-mediated codelivery of Curcumin and Chrysin: Possible application in prevention of breast cancer local recurrence. J Drug Deliv Sci Technol, 55, 101402.

Rasouli S, Zarghami N (2018). Synergistic growth inhibitory effects of chrysin and metformin combination on breast cancer cells through hTERT and cyclin D1 suppression. Asian Pac J Cancer Prev, 19, 977.

Samadzadeh S, Mousazadeh H, Ghareghomi S, et al (2021). In vitro anticancer efficacy of Metformin-loaded PLGA nanofibers towards the post-surgical therapy of lung cancer. J Drug Deliv Sci Technol, 61, 102318.

Serati-Nouri H, Jafari A, Roshangar L, et al (2020). Biomedical applications of zeolite-based materials: A review. Mater. Sci Eng $C, \mathbf{1 1 6}, 111225$.

Si L, Fu J, Liu W, et al (2020). Silibinin inhibits migration and invasion of breast cancer MDA-MB-231 cells through induction of mitochondrial fusion. Mol Cell Biochem, 463, 189-201.

Tavsan Z, Kayali HA (2019). Flavonoids showed anticancer effects on the ovarian cancer cells: Involvement of reactive oxygen species, apoptosis, cell cycle and invasion. Biomed Pharm, 116, 109004.

Wang B, Yuan F (2018). The association between estrogen receptor alpha gene rs 746432 polymorphism and the risk of breast cancer. Breast Cancer Res Treat, 172, 505.

Yang S, Traore Y, Jimenez C, Ho EA (2019). Autophagy induction and PDGFR- $\beta$ knockdown by siRNA-encapsulated nanoparticles reduce chlamydia trachomatis infection. Sci Rep, 9, 1-14.

Zappavigna S, Vanacore D, Lama S, et al (2019). Silybin-induced 
Ali Pourgholi et al

apoptosis occurs in parallel to the increase of ceramides synthesis and miRNAs secretion in human hepatocarcinoma cells. Int J Mol Sci, 20, 2190.

Zhang Y, Ge Y, Ping X, et al (2018). Synergistic apoptotic effects of silibinin in enhancing paclitaxel toxicity in human gastric cancer cell lines. Mol Med Rep, 18, 1835-41.

Zheng N, Liu L, Liu W-w, et al (2017). Crosstalk of ROS/RNS and autophagy in silibinin-induced apoptosis of MCF-7 human breast cancer cells in vitro. Acta Pharmacol Sin, 38, 277-89.

\section{๑๐०}

This work is licensed under a Creative Commons AttributionNon Commercial 4.0 International License. 